\title{
Proliferation of syndromes and acronyms in paediatric critical care: are we more or less confused?
}

\author{
$\mathrm{KL} \mathrm{Hon}^{1}$ *, MB, BS, MD, Alexander KC Leung ${ }^{2}$, FRCP(UK), FRCPCH, Jeff CP Wong ${ }^{1}$, MB, BS, MRCPCH \\ ${ }^{1}$ Department of Paediatrics and Adolescent Medicine, The Hong Kong Children's Hospital, Hong Kong \\ ${ }^{2}$ Department of Pediatrics, University of Calgary and Alberta Children's Hospital, Calgary, Canada
}

* Corresponding author: ehon@hotmail.com

Hong Kong Med J 2020;26:260-2

https://doi.org/10.12809/hkmj198059

As with many disciplines in medicine, syndromes, abbreviations, and acronyms have been coined to aid diagnosis and prognostication in paediatric intensive or critical care medicine (CCM). Although these acronyms are commonly used, they remain controversial. In clinical practice, CCM syndromes are clinical patterns resulting from the interaction between insult and host response. They are closely correlated to organ system failures and are common presentations of diverse aetiologic factors. Therefore, it is important to search for underlying aetiologies and subtypes. In research, the role of acronyms and syndromes is limited in epidemiological studies and clinical trials enrolling patients meeting syndrome

TABLE. Selected acronyms used in critical care medicine setting and their ICD codes

\begin{tabular}{lcc}
\hline Acronym & ICD-9-CM code & ICD-10-CM code \\
\hline ARDS & 518.82 & J80 \\
COVID-19 & & U07.1 \\
GVHD & 279.5 & D89.81 \\
MERS & & \\
MODS & 995.2 & $\mathrm{R} 65.2$ \\
\hline Pneumonia due to COVID-19 & & $\mathrm{J} 12.89$ \\
PRES & & $\mathrm{I} 67.83$ \\
SARS & 079.82 & $\mathrm{~B} 97.21$ \\
Severe sepsis & & $\mathrm{R} 65.2$ \\
SIRS & & $\mathrm{R} 65.1$ \\
SSSS & 695.81 & $\mathrm{~L} 00$ \\
\hline TLS & 277.88 & $\mathrm{E} 88.3$ \\
TMA & 446.6 & $\mathrm{M} 31.1$ \\
\hline TSS & 040.82 & $\mathrm{~A} 48.3$ \\
VOD & 573.8 & $\mathrm{~K} 76.5$ \\
\hline
\end{tabular}

Abbreviations: ARDS = acute respiratory distress syndrome; COVID-19 = coronavirus disease 2019; GVHD = graft-versus-host disease; ICD = International Classification of Diseases; MERS = Middle East respiratory syndrome; MODS = multiple organ dysfunction syndrome; PRES = posterior reversible encephalopathy syndrome; SARS = severe acute respiratory syndrome; SIRS = severe inflammatory response syndrome; SSSS = staphylococcal scald skin syndrome; TLS = tumour lysis syndrome; TMA = thrombotic microangiopathy;TSS = toxic shock syndrome; $\mathrm{VOD}=$ venoocclusive disease criteria, unless underlying subtypes and aetiologies are considered. As a result, study outcomes are often negative. Hence, we advocate that both syndrome codes and underlying aetiologic diagnoses be provided in International Classification of Diseases (ICD) coding to record prognostic and therapeutic significance (Table). Last, non-standardised acronyms contribute to confusion and should be used sparingly. Herein, we review some examples of syndromes and acronyms in use in CCM settings and consider whether their use is justified.

Acute respiratory distress syndrome (ARDS) is an important cause for CCM admission. ${ }^{1,2}$ Ashbaugh et $\mathrm{al}^{3}$ first described ARDS as an adulttype respiratory distress for a group of patients with progressive respiratory failure, refractory hypoxaemia, decreased functional residual capacity and lung compliance, and diffuse infiltration on chest radiography. In 1994, the American-European Consensus Conference introduced ARDS as a disease with acute-onset hypoxaemia, $\mathrm{PaO}_{2} / \mathrm{FiO}_{2}$ ratio $\leq 200$, bilateral infiltrates on chest radiographs, and the absence of left atrial hypertension. ${ }^{4}$ The international consensus criteria for ARDS were updated in 2012 and are known as the Berlin definition. ${ }^{5}$ However, ARDS is not a specific disease entity; it is a clinical syndrome which may be triggered by various pathologies such as trauma, pneumonia, and sepsis. As with many other syndromes, the term essentially describes non-cardiogenic pulmonary oedema of various aetiologies.

Acute respiratory distress syndrome also occurs in children, and is no longer restricted to adults. ${ }^{6}$ In 2015, the Paediatric Acute Lung Injury Consensus Conference proposed a definition for paediatric ARDS. ${ }^{7}$ This definition identified more patients with ARDS than did the Berlin criteria, but there were no differences in clinical outcomes. ${ }^{8}$ However, the Berlin definition offers no room for stratifying and identifying true ARDS patients because there is no re-evaluation of hypoxaemia under standard ventilator setting in a specific time period. ${ }^{9}$

The current (2015) ICD code for ARDS is 
518.82. This code is seldom used in paediatric hospital records in Hong Kong because many cases are diagnosed as pneumonia rather than ARDS. As a result, the hospital admission database might not accurately reflect the epidemiology of the disease in Hong Kong. ${ }^{10}$

Acute lung injury (ALI) was previously considered as a mild form of ARDS with $\mathrm{PaO}_{2} / \mathrm{FiO}_{2}$ of 200 to $300 \mathrm{mg} \mathrm{Hg}$ in the setting of a wedge pressure $<18 \mathrm{mg} \mathrm{Hg}$, bilateral infiltrates radiographically consistent with pulmonary oedema, and no clinical evidence of cardiac failure. It follows that patients with ARDS have ALI. However, the acronym has fallen into disuse and has been removed from Berlin definition to minimise confusion. ${ }^{11}$

In 2003, the World Health Organization (WHO) coined a new term severe acute respiratory syndrome (SARS) for an outbreak of pneumonitis that was later found to be a novel coronavirus pneumonia and a form of ARDS. ${ }^{12}$ Subsequently, there was an outbreak of acute pneumonitis due to another coronavirus in the Middle East, which the WHO coined severe acute respiratory illness (SARI) and later changed to Middle East respiratory syndrome (MERS). ${ }^{12}$ These acronyms (SARI and MERS) are widely used but essentially represent a form of ARDS or atypical pneumonia causing ARDS, making them unnecessary and potentially confusing. ${ }^{13,14}$ Some of these outbreaks were not so severe or had extra-respiratory symptoms, rendering the term SARS equivocal. No further novel syndromes or acronyms for ARDS have been coined by WHO to date. Nevertheless, COVID-19 has been coined to represent the current outbreak of coronavirus disease that started in December 2019. Most cases are mild but there have been patients who died of respiratory failure and ARDS. ${ }^{12}$

Multiple organ dysfunction syndrome (MODS), also known as multiple organ failure, total organ failure, or multisystem organ failure, refers to altered organ function in an acutely ill patient requiring medical intervention to achieve homeostasis. ${ }^{15,16}$ The definition of MODS is less controversial than that of ARDS, and MODS is also applied in paediatric CCM settings. Typically, MODS results from infection, accident, surgery, hypoperfusion, and hypermetabolism. The primary underlying aetiology triggers an uncontrolled inflammatory response. Sepsis is the most common cause of MODS. The altered organ function in patients with MODS is such that homeostasis cannot be maintained without intervention, and typically involves two or more organ systems. ${ }^{15}$ Management of MODS is mostly supportive, primarily maintaining adequate tissue oxygenation. The current ICD-10-CM code for MODS is 995.92. Prognosis is proportionate to the number of organ systems involved and is worse if cardiopulmonary and neurologic involvements are present. Mortality varies from $30 \%$ to $100 \%$ where the chance of survival is diminished as the number of organs involved increases.

Sepsis is a major disease in CCM settings. ${ }^{17}$ Various grading terms have been used such as sepsis, severe inflammatory response syndrome (SIRS), severe sepsis, septic shock, and recalcitrant septic shock. ${ }^{18}$ Confirmation of an aetiological pathogen is not required, and SIRS can be easily defined using physiological parameters only. The current 2019 ICD-10-CM code for SIRS is R65.1 for that of noninfectious origin and R65.11 for that with acute organ dysfunction. Prognosis for SIRS is based on the underlying diagnosis and co-morbidities. The Third International Consensus Definitions for Sepsis and Septic Shock (Sepsis-3) criteria define septic shock as a subset of sepsis in which particularly profound circulatory, cellular, and metabolic abnormalities are associated with a greater risk of mortality than with sepsis alone. ${ }^{16}$ Patients with septic shock can be clinically identified by a vasopressor requirement to maintain a mean arterial pressure of $\geq 65 \mathrm{~mm} \mathrm{Hg}$ and serum lactate level $>2 \mathrm{mmol} / \mathrm{L}(>18 \mathrm{mg} / \mathrm{dL})$ in the absence of hypovolaemia. ${ }^{16}$ Many cases of paediatric sepsis are underdiagnosed and likely coded in hospital records in alternative diagnoses such as febrile seizure, encephalitis, urinary tract infection, or myocarditis.

Toxic shock syndrome (TSS) and staphylococcal scald skin syndrome (SSSS) are sepsis syndromes that are used only occasionally. ${ }^{19,20}$ Bacterial toxins such as the streptococcal or staphylococcal enterotoxins are implicated in their pathogenesis. ${ }^{21}$ Prognosis depends mainly on the promptness in diagnosis and treatment instituted. However, neither the organism nor the toxins (eg toxic shock syndrome toxin 1) are commonly or routinely isolated, therefore limiting the usefulness of these terminologies. In paediatrics, there have been keen proliferations of novel acronyms in the recent COVID-19 pandemic due to the virus SARS-CoV-2. Paediatric multisystem inflammatory syndrome (PMIS), multisystem inflammatory syndrome in children (MIS-C), and paediatric inflammatory multisystem syndrome temporally associated with SARS-CoV-2 (PIM-TS) are all recently coined acronyms for a systemic disease involving persistent fever, inflammation, and organ dysfunction following exposure to SARS-CoV-2. This syndrome has been considered to resemble Kawasaki disease and SIRS and associated with the hyperinflammation in cytokine release syndrome and cytokine storm syndrome. ${ }^{22}$

\section{Conclusion}

The acronym syndromes encountered in CCM settings are descriptive terminologies that should remain simple in definition. However, such 
syndromes may not necessarily aid diagnosis and prognostication. Users of these acronyms must understand the limitations and confusions behind these terminologies. Terms such as SARI, ALI, TSS, and SSSS are used infrequently and should be eliminated to avoid confusion. The underlying aetiologies of any syndrome must be explored and treated. For disease coding and prognostication analyses, all relevant aetiological factors must be considered.

\section{Author contributions}

All authors contributed to the concept of the study, acquisition and analysis of the data, drafting of the manuscript, and critical revision of the manuscript for important intellectual content. All authors had full access to the data, contributed to the study, approved the final version for publication, and take responsibility for its accuracy and integrity.

\section{Conflicts of interest}

As an editor of the journal, KL Hon was not involved in the peer review process. Other authors declare that they have no other conflict of interest.

\section{Funding/support}

This commentary received no specific grant from any funding agency in the public, commercial, or not-for-profit sectors.

\section{References}

1. Dellinger RP, Levy MM, Carlet JM, et al. Surviving sepsis campaign: international guidelines for management of severe sepsis and septic shock: 2008. Crit Care Med 2008;36:296-327.

2. Cornfield DN. Acute respiratory distress syndrome in children: physiology and management. Curr Opin Pediatr 2013;25:338-43.

3. Ashbaugh DG, Bigelow DB, Petty TL, Levine BE. Acute respiratory distress in adults. Lancet 1967;2:319-23.

4. Bernard GR, Artigas A, Brigham KL, et al. The AmericanEuropean Consensus Conference on ARDS. Definitions, mechanisms, relevant outcomes, and clinical trial coordination. Am J Respir Crit Care Med 1994;149(3 Pt 1):818-24.

5. ARDS Definition Task Force, Ranieri VM, Rubenfeld GD, et al. Acute respiratory distress syndrome: the Berlin Definition. JAMA 2012;307:2526-33.

6. Li CY, Luk MP, Hon KL. Paediatric acute respiratory distress syndrome: a review of recent advances in management. J Paediatr Respirol Crit Care 2016;12:4-9.

7. The Pediatric Acute Lung Injury Consensus Conference Group. Pediatric acute respiratory distress syndrome: consensus recommendations from the Pediatric Acute Lung Injury Consensus Conference. Pediatr Crit Care Med 2015;16:428-39.

8. Gupta S, Sankar J, Lodha R, Kabra SK. Comparison of prevalence and outcomes of pediatric acute respiratory distress syndrome using pediatric acute lung injury consensus conference criteria and berlin definition. Front Pediatr 2018;6:93.

9. Villar J, Kacmarek RM. The American-European Consensus Conference definition of the acute respiratory distress syndrome is dead, long live positive end-expiratory pressure! Med Intensiva 2012;36:571-5.

10. To KK, Hung IF, Li IW, et al. Delayed clearance of viral load and marked cytokine activation in severe cases of pandemic H1N1 2009 influenza virus infection. Clin Infect Dis. 2010;50:850-9.

11. López-Fernández Y, Azagra AM, de la Oliva P, et al. Pediatric acute lung injury epidemiology and natural history study: incidence and outcome of the acute respiratory distress syndrome in children. Crit Care Med 2012;40:3238-45.

12. Hon KL, Leung KK. Severe acute respiratory symptoms and suspected SARS again 2020. Hong Kong Med J 2020;26:78-9.

13. Hon KL, Li AM, Cheng FW, Leung TF, Ng PC. Personal view of SARS: confusing definition, confusing diagnoses. Lancet 2003;361:1984-5.

14. Hon KL. MERS = SARS? Hong Kong Med J 2015;21:478.

15. Ames SG, Workman JK, Olson JA, et al. Infectious etiologies and patient outcomes in pediatric septic shock. J Pediatric Infect Dis Soc 2017;6:80-6.

16. Singer M, Deutschman CS, Seymour CW, et al. The third international consensus definitions for sepsis and septic shock (Sepsis-3). JAMA 2016;315:801-10.

17. Tusgul S, Carron P-N, Yersin B, Calandra T, Dami F. Low sensitivity of qSOFA, SIRS criteria and sepsis definition to identify infected patients at risk of complication in the prehospital setting and at the emergency department triage. Scand J Trauma Resusc Emerg Med 2017;25:108.

18. Rhee C, Klompas M. New sepsis and septic shock definitions: clinical implications and controversies. Infect Dis Clin North Am 2017;31:397-413.

19. Resnick SD. Staphylococcal toxin-mediated syndromes in childhood. Semin Dermatol 1992;11:11-8.

20. Leung AK, Barankin B, Leong KF. Staphylococcal-scalded skin syndrome: evaluation, diagnosis, and management. World J Pediatr 2018;14:116-20.

21. Leung TN, Hon KL, Leung AK. Group A Streptococcus disease in Hong Kong children: an overview. Hong Kong Med J 2018;24:593-601.

22. Hon KL, Leung KK, Leung AK, et al. Overview: The history and pediatric perspectives of severe acute respiratory syndromes: novel or just like SARS. Pediatr Pulmonol 2020 Jun 1. Epub ahead of print. 